\title{
Examining mitigation schemes for synchrotron radiation in high-energy hadron colliders
}

\author{
G. Guillermo, ${ }^{1,2, *}$ D. Sagan, ${ }^{3}$ and F. Zimmermann ${ }^{2}$ \\ ${ }^{1}$ Departamento de Física Aplicada, Cinvestav del IPN, Unidad Mérida, A.P. 73 Cordemex, \\ 97310 Mérida, Yucatán, Mexico \\ ${ }^{2}$ CERN, BE Department, 1211 Geneva 23, Switzerland \\ ${ }^{3}$ Cornell Laboratory for Accelerator-based Sciences and Education, Cornell University, \\ Ithaca, New York 14850, USA
}

(Received 4 December 2017; published 14 February 2018)

\begin{abstract}
At high proton-beam energies, beam-induced synchrotron radiation is an important source of heating, of beam-related vacuum pressure increase, and of primary photoelectrons, which can give rise to an electron cloud. We use the Synrad3D code developed at Cornell to simulate the photon distributions in the arcs of several existing, planned, or proposed highest-energy hadron colliders to analyze the efficiency of several techniques developed, or proposed, to mitigate the negative effects of synchrotron radiation, such as a sawtooth surface and slots in the beam screen.
\end{abstract}

DOI: $10.1103 /$ PhysRevAccelBeams.21.021001

\section{INTRODUCTION}

At high proton-beam energies, beam-induced synchrotron radiation is an important source of heating, of beam-related vacuum pressure increase, and of primary photoelectrons, which can give rise to an electron cloud [1-7].

The simulation code Synrad3D developed at Cornell [8] generates and tracks synchrotron-radiation photons in an accelerator beam line, including specular and diffuse reflection on the chamber surface. The photons are generated randomly in any bending field, with the initial parameters determined by the local beam distribution, the local electromagnetic field, and the beam energy. When a photon hits the chamber wall, its reflection probability depends on the energy and angle of incidence, as well as on the material, including combinations of multiple layers, and on the surface roughness. As one option, reflection tables provided by the Lawrence Berkeley National Laboratory Center for X-Ray Optics [9] can be employed. The simulations reported in the following will make use of these tables.

An earlier tracking code for synchrotron radiation, called PHOTON [10], considered only a generic reflection coefficient, which was applied independently of the photon energy and incidence angle.

\section{*Gerardo.Guillermo.Canton@ cern.ch}

Published by the American Physical Society under the terms of the Creative Commons Attribution 4.0 International license. Further distribution of this work must maintain attribution to the author(s) and the published article's title, journal citation, and DOI.
A first approach to this problem using the new tool Synrad3D was presented in Ref. [11] and a second one in Ref. [12].

We are now deploying Synrad3D to simulate the photon distributions in the arcs of various high-energy circular hadron colliders (LHC [13], HL-LHC [14], HE-LHC [15], and FCC-hh). Specifically, for the LHC we study the effect of the sawtooth pattern imprinted on the vacuum chamber; for the HL-LHC, the consequences of the achromatic telescopic squeeze (ATS) optics [16] with large beta beating in the arcs; for the FCC-hh, the effect of a novel beamscreen design, with a long slit surrounded by a "folded" antechamber [17]; and finally, for HE-LHC, we analyze two proposed options for the vacuum chamber.

\section{A. Modeling the diffuse reflection}

The model used by Synrad3D for simulating diffuse reflections was developed by Dugan and Sagan [8]. The model gives the probability $P(x, \phi)$ of scattering a photon diffusely, where $x=\cos (\theta)$ and $(\theta, \phi)$ are the polar angles of the scattered outgoing photon with $\phi=0$ indicating that the scattered photon's velocity vector is in the plane formed by the incoming photon's velocity vector and the surface normal. Generally, the scattering probability $P(x, \phi)$ from a rough surface depends on the rms surface roughness $\sigma$, the photon wavelength $\lambda$, and the incident photon's angle, as well as the atomic properties of the surface.

For calculational convenience, instead of working with the probability function $P(x, \phi)$, which is a function of two variables, two one-dimensional probability functions are used. The first function is $P_{x}(x)$, which is $P(x, \phi)$ integrated over $\phi$ [Eq. (A140) in Ref. [8]]. For a given 
scattering event, $P_{x}(x)$ is first used, with the help of a random number generator, to choose a value for $x$. After this, the probability function $P(\phi \mid x)$, which is the probability of scattering at an angle $\phi$ for a given $x$, is used to choose a value for $\phi$.

For a typical technical vacuum chamber surface, the rms surface roughness is of the order of $\sigma \sim 200 \mathrm{~nm}$ [8]. Given the low surface roughness used for our simulations $(\sigma=50 \mathrm{~nm})$ [18], the probability function $P_{x}(x)$ becomes highly peaked where the reflected polar angle $x$ equals the incident polar angle. That is, the diffuse reflection distribution resembles the delta function distribution for specular reflections [8]. In prior simulations, a 30th-order Chebyshev fit to $P_{x}(x)$ was used to integrate $P_{x}(x)$ (the integration is needed to normalize the scattering probability). With the low surface roughness of the present simulations, this proved to be a poor fit due to the deltafunction-like nature of $P_{x}(x)$. To address this issue, the Chebyshev fit was replaced by an adaptive Akima spline fit [19], which better represents extremely peaked functions for a number of reasons. The Akima spline has the advantage of locality in that the calculated slope at a knot point is affected only by the neighboring knot points. Additionally, the Akima spline does not mandate a continuous second derivative at the knot points. This is an advantage with highly peaked functions, since, in this case, there are large changes in the second derivative. Finally, the adaptive point selection places knots points to minimize the estimated error in integrated area. With a peaked function, more knots will be used near the peak where the second derivative is changing the most. This nonuniform distribution of knots minimizes the computation time needed.

\section{LHC}

The Large Hadron Collider (LHC) at CERN presently collides two proton beams with an energy of $6.5 \mathrm{TeV}$ each. At the design beam energy of $7 \mathrm{TeV}$, the synchrotron radiation heat load amounts to $0.17 \mathrm{~W} / \mathrm{m}$ per aperture.

In the LHC cold arcs, the synchrotron radiation is intercepted by a beam screen at a temperature of 4.6$19 \mathrm{~K}$, e.g., higher than the $1.9 \mathrm{~K}$ of the cold bore [13]. The beam screen is made from stainless steel with a $75 \mu \mathrm{m}$ copper coating. The total thickness of the beam screen is $1.08 \mathrm{~mm}$ including the copper layer [20].

Some example reflection probabilities for an LHC-like chamber surface with a 10-nm-thick carbon layer on top of the copper are presented in Fig. 1. The carbon layer models the effect of surface conditioning due to electron bombardment ("electron-cloud scrubbing") [21]. For protons of $7 \mathrm{TeV}$ energy passing through an arc bending magnet, the critical photon energy for LHC proton synchrotron radiation is about $44 \mathrm{eV}$. To reduce photon reflections, the inner surface of the beam screen, on the horizontally outward side, features a sawtooth pattern, which should ensure an almost perpendicular impact. The sawtooth has a

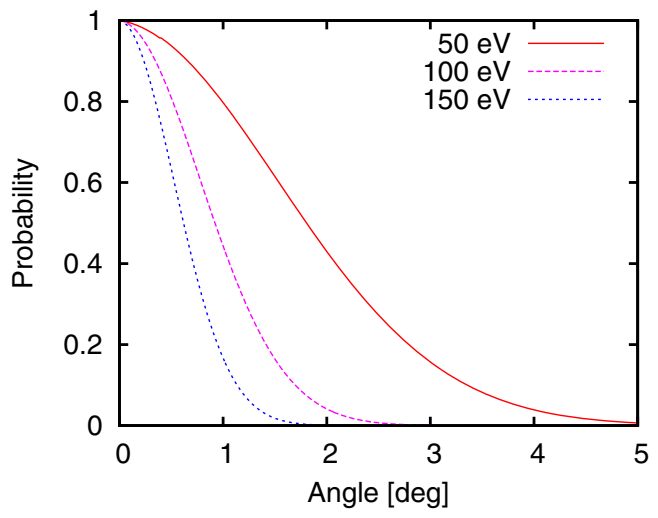

FIG. 1. Specular photon reflectivity as a function of the angle of incidence for several different photon energies, considering a $10 \mathrm{~nm}$ carbon layer on top of a copper surface and $50 \mathrm{~nm} \mathrm{rms}$ surface roughness.

longitudinal period of about $500 \mu \mathrm{m}$ and a horizontal amplitude of around $35 \mu \mathrm{m}$. The vertical extent of the sawtooth pattern is $\pm 7.5 \mathrm{~mm}$ from the equatorial plane [20]. This vacuum chamber is shown in Fig. 2.

Since 2008, a number of LHC arc beam screens have been installed with the wrong orientation ("inverted sawtooth") [23]. Figure 3 shows the model of the LHC inverted sawtooth beam screen implemented in Synrad3D.

Simulated distributions of absorbed photons in Fig. 4 and the corresponding reflection distributions in Fig. 5 illustrate the effect of the sawtooth surface as well as the effect of an inverted sawtooth. The number of passages is defined as the number of reflections plus one.

For the case of the sawtooth, most photons are absorbed at the moment and location of primary impact $(\phi \approx 0)$, as the mean number of passages is about 1 , or the mean number of reflections close to 0 , in Fig. 5. This explains the peak of the absorbed photon distribution at $\phi \approx 0$, in Fig. 4 .

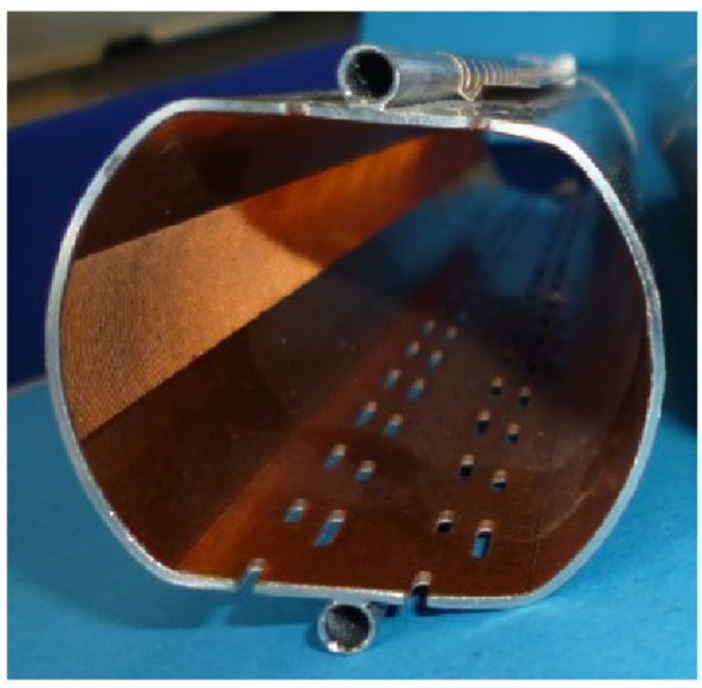

FIG. 2. Cross section of the LHC vacuum chamber [22]. 
(a)

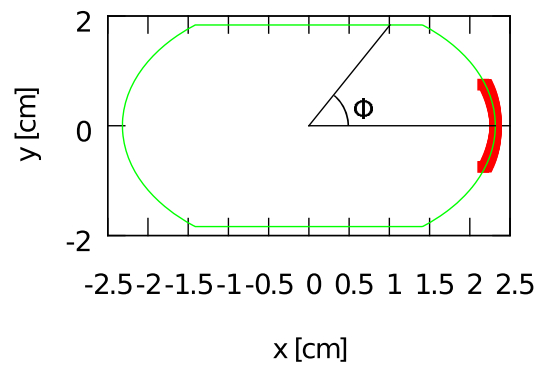

(c)

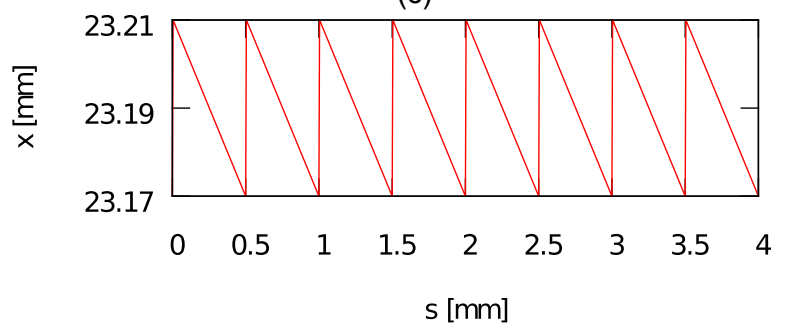

Sawtooth (b)

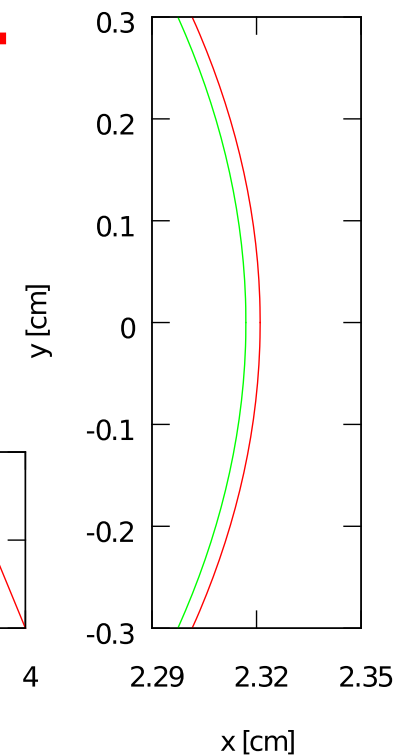

FIG. 3. Synrad3D model of the LHC beam-screen design. (a) $x y$ cross section, (b) enlarged $x y$ cross section, (c) $x s$ cross section. If the beam is moving towards the positive $s$ direction, it will see an inverted sawtooth; if it is moving towards negative $s$, it will see the correct form of the sawtooth.

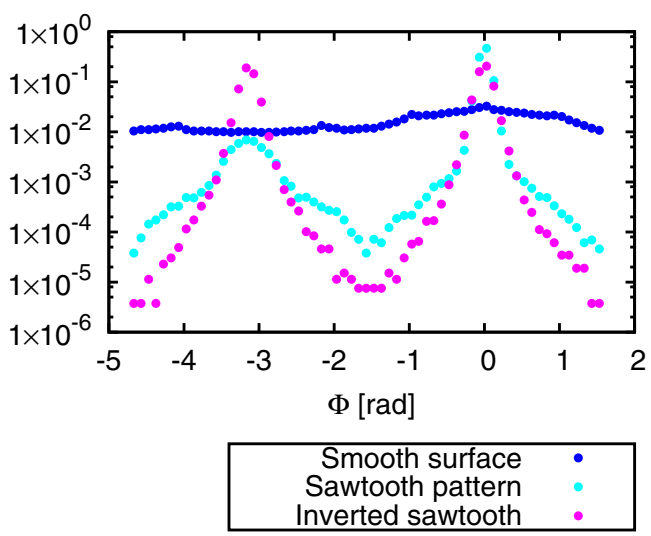

FIG. 4. Simulated azimuthal distribution (integral normalized to unity) of absorbed photons without (blue curve) and with a sawtooth (teal curve) and with an inverted sawtooth (purple curve) chamber.

In the case of the smooth chamber, the average number of reflections is higher than 80, in Fig. 5, and, as a result, the location of absorbed photons is almost uniform around the azimuth, in Fig. 4, with only a slight bias towards the primary impact area, and tiny local maxima corresponding to the transitions between circular and flat portions of the beam screen in Fig. 3 (top left).

Finally, for the inverted sawtooth, the average number of photon reflections is about two (Fig. 4), which gives rise to almost equally high absorption maxima on the horizontally outward and inward sides of the chamber in the horizontal plane, as can be seen in Fig. 4. With this rather low number of reflections, photons are not yet spread towards the top and bottom of the vacuum chamber.

With the sawtooth, only $0.2 \%$ each of the photons are absorbed on the top and bottom surfaces, whereas $99.6 \%$ are absorbed on the sides as is shown in Fig. 6.

By contrast, with no sawtooth, $41 \%$ of the photons are absorbed on the primary impact side, $23 \%$ on the opposite side, and $18 \%$ each on the top and bottom of the chamber. These results can be compared with experimental measurements at the VEPP-2M electron-positron collider at the Budker Institute of Nuclear Physics in Novosibirsk, Siberia, Russia [24,25] and the ELETTRA synchrotron research center in Trieste, Italy [26].

For a quantitative comparison, the VEPP-2M measurement for a smooth copper coated surface without a sawtooth at 20 mrad grazing incidence revealed a photon forward reflectivity $R$ of up to $95 \%$ [25]. This would correspond to an average number of $\langle n\rangle=(1-R) \sum_{n} R^{n-1}=1 /(1-R) \approx$ 20 photon passages through the chamber until absorption. Adding another 2\%-4\% diffusely reflected photons [25], the average number of photon passages in the measurements would be between 33 and 100, which is consistent with the value of about 80 found by the Synrad3D simulations, in the top picture in Fig. 5. For the sawtooth surface, a much lower total reflectivity of about $10 \%$ was measured [26]. This translates into an average number of passages not much above 1 , and the Synrad3D simulations also predict a value barely above 1 (see the bottom picture in Fig. 5).

The almost tenfold reduction of photons hitting the top and bottom of the chamber confirms the intended effect of the sawtooth structure, namely, to greatly reduce the number 

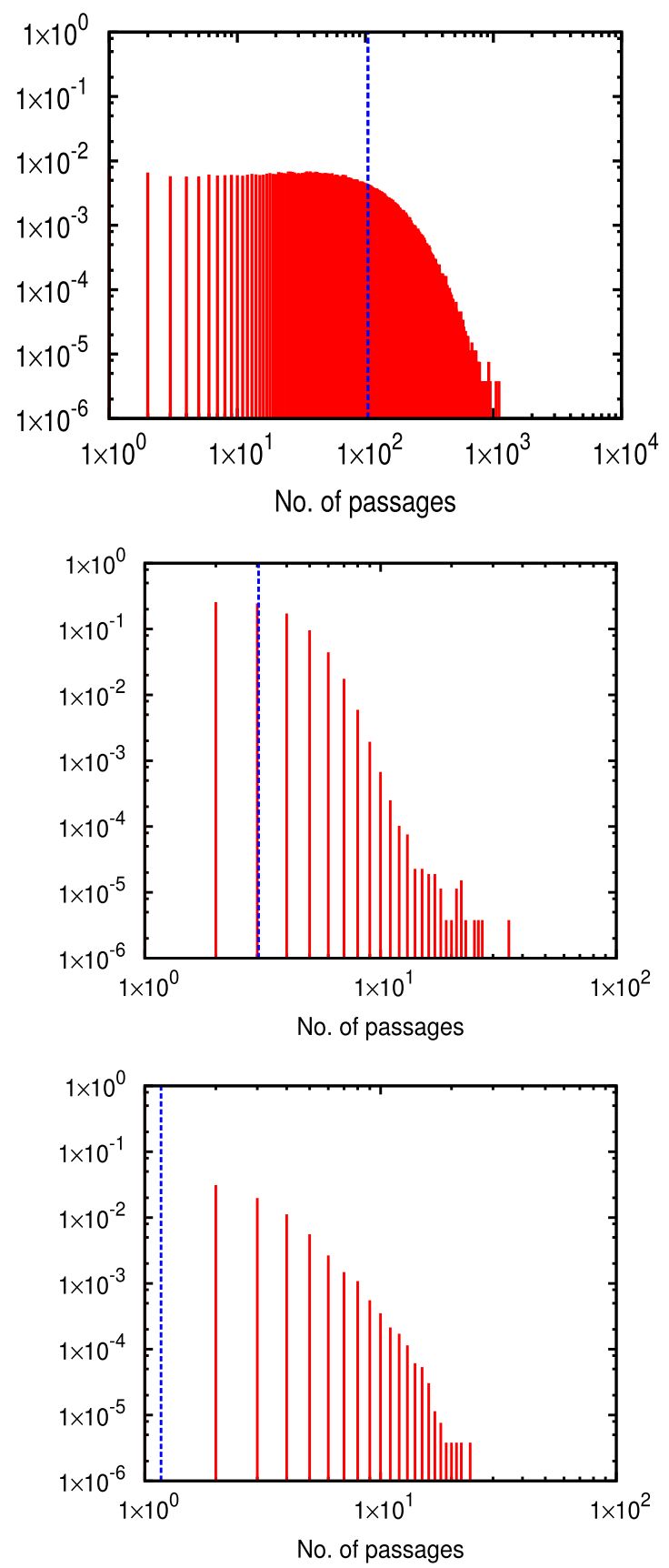

FIG. 5. Distribution of the number of photon passages till absorption without (top) and with a sawtooth chamber (bottom) and an inverted sawtooth (center). The blue line represents the mean number of passages.

of photoelectrons generated at the top and bottom of the vacuum chamber in the arc dipole magnets (from where they could approach the beam, following the vertical field lines, and contribute to further electron-cloud buildup).

While for the inverted sawtooth the average number of reflections is higher than for a correct sawtooth (Fig. 5), to our surprise the inverted sawtooth proves even more efficient than the sawtooth in reducing the

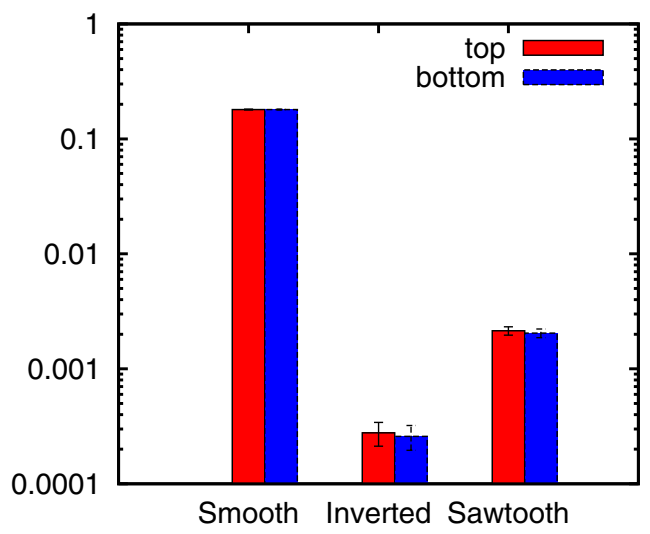

FIG. 6. Fraction of photons absorbed at the top and bottom of the vacuum chamber of the LHC, with a $95 \%$ confidence interval.

number of photons absorbed at the top or bottom of the chamber (Fig. 4). This strongly suggests that the assumptions for an inverted sawtooth made in Ref. [27] were very pessimistic.

\section{A. HL-LHC}

The High-Luminosity LHC (HL-LHC) is an approved upgrade of the LHC, to be implemented around the year 2025, which aims at a tenfold increase in the total integrated luminosity of the LHC.

Aside from an almost 2 times higher beam current, which will approximately double the photon flux in the arcs, the main difference between the LHC [13] and HL-LHC [14] is a beta wave intentionally introduced through the adjacent arcs to squeeze the $\beta^{*}$ at two highluminosity collision points. This optics scheme is called the ATS [16]. It exists in round $\left(\beta_{x}^{*}=\beta_{y}^{*}\right)$ and flat configurations $\left(\beta_{x}^{*} \gg \beta_{y}^{*}\right)$. With a large vertical beta beat in the LHC arcs " 45 " and " 56 ," the distribution of photons hitting the chamber wall (and being absorbed there) may change. For example, in the "presqueeze" optics with $\beta_{x, y}^{*}=0.44 \mathrm{~m}$, the minimum beta function in the arc is $32 \mathrm{~m}$, whereas for a nonbaseline flat ATS optics with $\beta_{y}^{*}=$ $0.05 \mathrm{~m}$ in IP5 [28], the minimum vertical beta function shrinks to $\beta_{y, \min } \approx 16 \mathrm{~m}$ in $\operatorname{arcs} 45$ and 56 . This is to be contrasted with the baseline flat ATS optics, which has a vertical beta function in IP5 of $0.075 \mathrm{~m}$ [29]. Nevertheless, with a geometric rms emittance $\varepsilon_{y}$ of about $0.3 \mathrm{~nm}$, the corresponding maximum rms divergence of $\sigma_{y^{\prime}, \max } \approx$ $\left(\varepsilon_{x} / \beta_{y, \text { min }}\right)^{1 / 2} \approx 5 \times 10^{-6}$ is still small compared with the rms angle of the photons emitted, $\sim \gamma^{-1} \approx 1.3 \times 10^{-4}$. Therefore, no large effect of the ATS optics on the photon distribution is expected. This expectation was confirmed in Synrad3D simulations by comparing photon distributions for a squeezed flat optics with those for the presqueeze (equal to the standard LHC arc optics) [12]. 


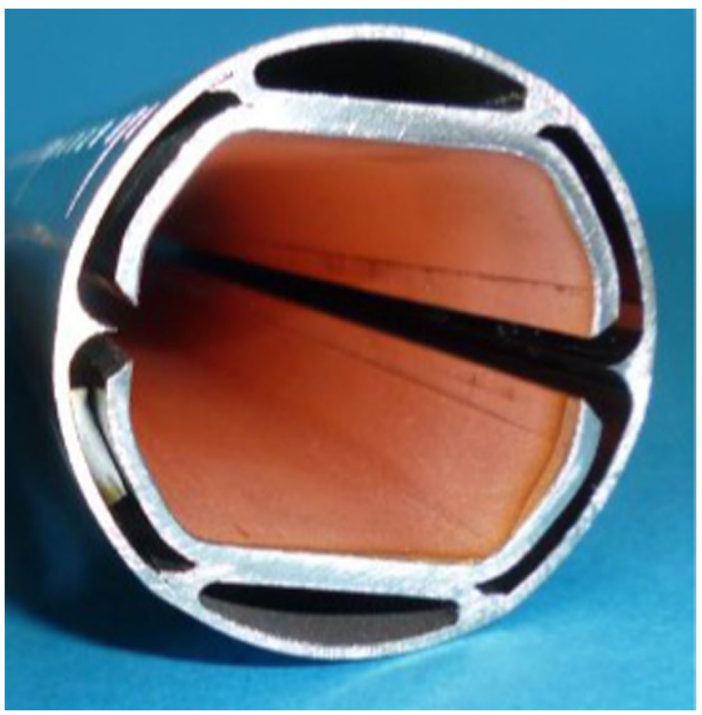

FIG. 7. Cross section of the proposed FCC-hh vacuum chamber [22].

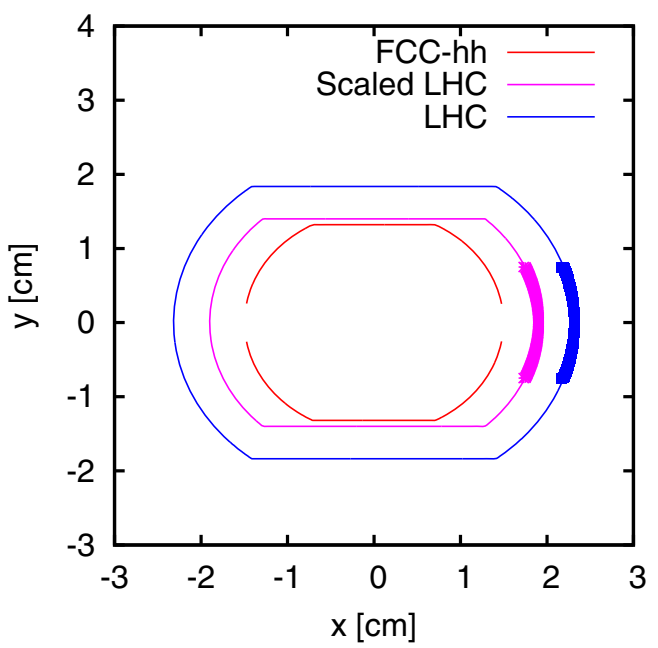

FIG. 8. Vacuum chamber cross sections for FCC-hh, LHC, and scaled LHC chambers. The thicker line represents the sawtooth on the wall, and the transparent region is an opening slot (modeled as a perfect absorber).

\section{FCC-hh}

A proposed Future Circular Hadron Collider (FCC-hh), providing proton collisions at a center-of-mass energy of $100 \mathrm{TeV}$, would utilize novel $16 \mathrm{~T}$ bending magnets, based on the $\mathrm{Nb}_{3} \mathrm{Sn}$ superconductor, and be installed in a new $\sim 100 \mathrm{~km}$ tunnel near Geneva [30-32]. In the collider arcs, the $50 \mathrm{TeV}$ proton beams would emit an average synchrotron radiation power of $28 \mathrm{~W} / \mathrm{m}$ per aperture, which is roughly 100 times higher than the corresponding figure for the HL-LHC. The critical photon energy is above $4 \mathrm{keV}$, also 100 times higher than for the LHC. A new type of higher-temperature beam screen [22] inside the cold bore of

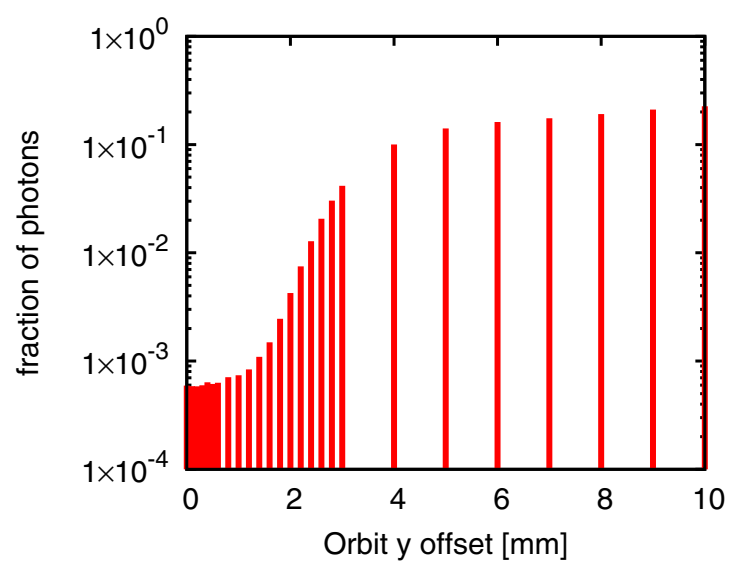

FIG. 9. Simulated fraction of photons absorbed on the inner FCC-hh beam screen as function of the peak vertical orbit error.

the arc magnets is intended to intercept this synchrotron radiation while minimizing the generation of an electron cloud in the beam pipe proper.

The FCC-hh beam-screen shape with an integrated compact antechamber is illustrated in Fig. 7. Slots in the equatorial plane with a vertical full height of $5 \mathrm{~mm}$ will absorb most of the photons, thereby facilitating the beamscreen cooling and stabilizing the beam vacuum [30,31]. As a first approximation, we model these slots as perfect absorbers as shown in Fig. 8.

The simulation considered two half cells of the FCC-hh arcs with a length of $213.89 \mathrm{~m}$. In a half cell, the main synchrotron radiation source is six dipole magnets, each $14.3 \mathrm{~m}$ long, with a dipole field of $16 \mathrm{~T}[30,31]$. A value of the normalized emittance of $\varepsilon=2.2 \mu \mathrm{m}$ is considered [32] and the energy equal to the collision beam energy $(50 \mathrm{TeV})$.

For a centered orbit, a fraction of $0.05 \%$ of the emitted photons are hitting the beam screen outside of the absorber slots. Figure 9 shows the dependence of this fraction on a vertical orbit offset, setting a tolerance on the acceptable closed-orbit distortions in the FCC-hh of about $1 \mathrm{~mm}$ (peak offset from the horizontal plane).

\section{HE-LHC}

The High-Energy LHC (HE-LHC) is a proposed $27 \mathrm{TeV}$ proton-proton collider in the existing LHC tunnel [15]. The HE-LHC can be realized by replacing the LHC's $8.33 \mathrm{~T} \mathrm{Nb}$-Ti dipole magnets with $16 \mathrm{~T} \mathrm{Nb}_{3} \mathrm{Sn}$ magnets developed for the FCC-hh. A high-quality beam available from the upgraded LHC injector complex together with significant radiation damping allows achieving a respectable integrated luminosity. On the other hand, the average arc synchrotron radiation power of $4.6 \mathrm{~W} / \mathrm{m}$ per aperture is much higher than for the LHC $(0.17 \mathrm{~W} / \mathrm{m}$ per aperture $)$ or HL-LHC $(0.33 \mathrm{~W} / \mathrm{m}$ per aperture). The critical photon energy in the arc bending magnets is about $300 \mathrm{eV}$ for the HE-LHC, to be compared with $44 \mathrm{eV}$ for the LHC optics. 


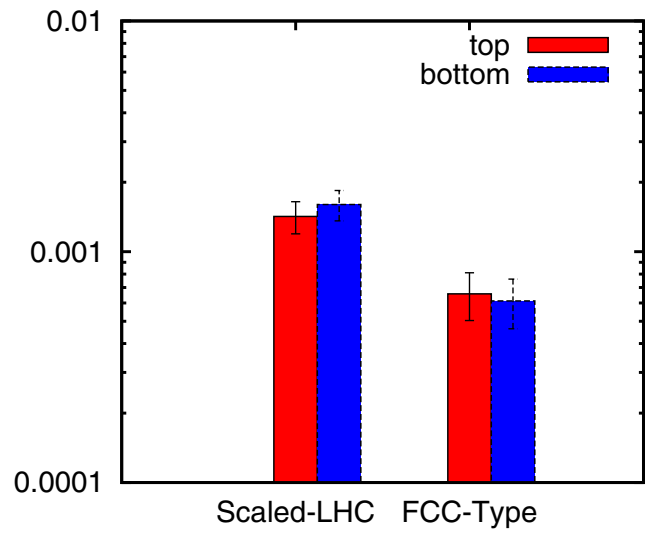

FIG. 10. Fraction of photons absorbed at the top and bottom of two proposed vacuum chambers for the HE-LHC, with a $95 \%$ confidence interval.

In the following, we consider, for the HE-LHC, the same optics as for the LHC.

For this circular collider, two options for the vacuum chamber were considered: an FCC-hh type or a scaled version of the LHC beam screen; both are shown in Fig. 8. Figure 10 illustrates that the FCC-hh chamber is 4 or 5 times more efficient than the scaled LHC chamber in reducing photon absorption at the top and the bottom of the chamber.

\section{ENERGY DEPENDENCE}

Since the angular distribution of the emitted synchrotron-radiation photons depends on the beam energy and emittance, we simulate, for different beam energies, the fraction of photons escaping from the slots of the FCC-hhtype beam screen and being absorbed on the surface of the

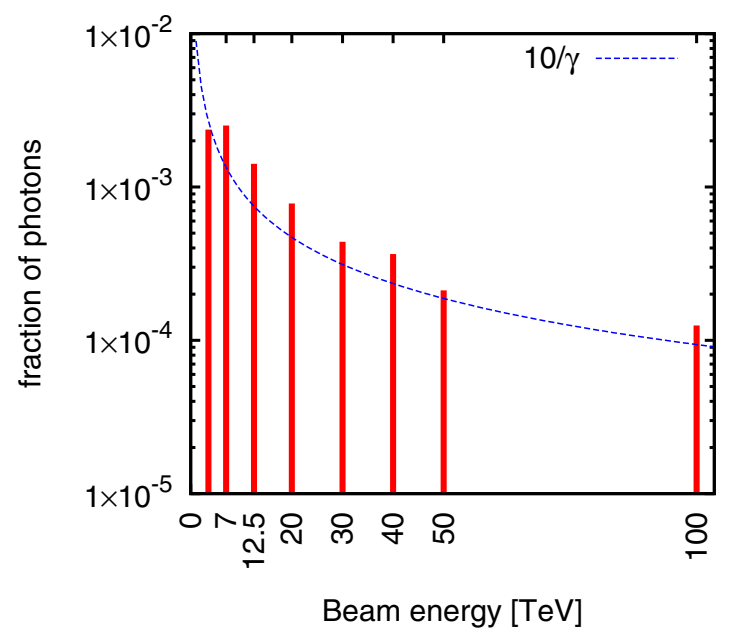

FIG. 11. Fraction of photons absorbed by an FCC-hh-type chamber as a function of the beam energy for a normalized emittance of $\varepsilon=2.5 \mu \mathrm{m}$ and the LHC optics. beam chamber proper. Considering the LHC (HE-LHC) optics as an example, the result of such an energy scan is displayed in Fig. 11. The FCC-hh beam screen is extremely effective at $50 \mathrm{TeV}$, but a factor of 10 less at $7 \mathrm{TeV}$. As indicated in the figure, the fraction of photons not entering the slots decreases roughly inversely with the beam energy, as the vertical opening angle of the synchrotron radiation.

\section{CONCLUSIONS}

We have modeled the synchrotron radiation flux in the arcs of four different hadron colliders, using the code Synrad3D developed at Cornell. The effect of electron-cloud surface conditioning ("scrubbing") was modeled by considering a thin $10 \mathrm{~nm}$ carbon layer on top of the beam screen's copper coating.

For the present LHC, the simulations demonstrate the efficiency of the sawtooth surface in reducing the average number of reflections almost to zero. Thereby, as intended, the sawtooth greatly decreases the number of photons absorbed at the top and bottom of the chamber, from where, in a dipole magnetic field, photoelectrons could approach the beam. Remarkably, our simulations also suggest that an inverted sawtooth, yielding an average number of reflections of about 2, might be even more efficient in this regard. This result could be attributed to a predominance of specular over diffuse reflection for the inverted sawtooth with extremely low surface roughness.

The ATS optics of the HL-LHC, with significant beta beating across the arcs, does not noticeably change the azimuthal photon distributions.

For the FCC-hh, a new type of beam screen is proposed. The Synrad3D simulations confirm that this beam screen significantly reduces the number of photons absorbed inside the beam chamber proper, by more than 2 orders of magnitude.

For the HE-LHC, the FCC-hh vacuum chamber is about 5 times more efficient than the scaled LHC chamber in reducing the number of photons absorbed at the top and bottom of the chamber and, therefore, in suppressing possible electron-cloud buildup due to photoemission.

\section{ACKNOWLEDGMENTS}

We thank Marton Ady, Vincent Baglin, Roberto Cimino, Guillermo Contreras, Massimo Giovannozzi, Roberto Kersevan, and Humberto Maury for valuable discussions and support. This work was supported, in part, by the European Commission under the HORIZON 2020 Integrating Activity project ARIES, Grant Agreement No. 730871, and by the Mexican CONACyT "BEAM" Program. Synrad3D development is supported by the U.S. National Science Foundation (PHY-0734867, PHY1002467, and PHY-1068662) and U.S. Department of Energy. 
[1] K. Ohmi, Beam-Photoelectron Interactions in Positron Storage Rings, Phys. Rev. Lett. 75, 1526 (1995).

[2] K. Ohmi and F. Zimmermann, Head-Tail Instability Caused by Electron Clouds in Positron Storage Rings, Phys. Rev. Lett. 85, 3821 (2000).

[3] G. Rumolo, F. Ruggiero, and F. Zimmermann, Simulation of the electron-cloud build up and its consequences on heat load, beam stability, and diagnostics, Phys. Rev. ST Accel. Beams 4, 012801 (2001).

[4] K. Ohmi, F. Zimmermann, and E. Perevedentsev, Wakefield and fast head-tail instability caused by an electron cloud, Phys. Rev. E 65, 016502 (2001).

[5] M. T. F. Pivi and M. A. Furman, Electron cloud development in the proton storage ring and in the spallation neutron source, Phys. Rev. ST Accel. Beams 6, 034201 (2003).

[6] F. Zimmermann, Review of single bunch instabilities driven by an electron cloud, Phys. Rev. ST Accel. Beams 7, 124801 (2004).

[7] E. Benedetto, G. Franchetti, and F. Zimmermann, Incoherent Effects of Electron Clouds in Proton Storage Rings, Phys. Rev. Lett. 97, 034801 (2006).

[8] G. Dugan and D. Sagan, Simulating synchrotron radiation in accelerators including diffuse and specular reflections, Phys. Rev. Accel. Beams 20, 020708 (2017).

[9] B. L. Henke, E. M. Gullikson, and J.C. Davis, X-ray interactions: Photoabsorption, scattering, transmission, and reflection at $e=50-30,000 \mathrm{ev}, z=1-92$, At. Data Nucl. Data Tables 54, 181 (1993).

[10] F. Zimmermann, CERN Technical Report No. LHCPROJECT-NOTE-237, 2000.

[11] G. H. I. Maury et al., Synchrotron-radiation photon distribution for highest energy circular colliders, in Proceedings of the 4th International Particle Accelerator Conference, IPAC-2013, Shanghai, China, 2013 (JACoW, Shanghai, China, 2013), p. 1340.

[12] G. Guillermo, D. Sagan, and F. Zimmermann, Simulating Proton Synchrotron Radiation in the Arcs of the LHC, HLLHC and FCC-hh, in Proceedings of the International Particle Accelerator Conference (JACoW, Busan, 2016), pp. 2073-2076.

[13] O. S. Brüning, P. Collier, P Lebrun, S. Myers, R. Ostojic, J. Poole, and P. Proudlock, LHC Design Report, CERN Yellow Reports: Monographs (CERN, Geneva, 2004).

[14] G. Apollinari, I. Béjar Alonso, O. Brüning, M. Lamont, and L. Rossi, High-Luminosity Large Hadron Collider (HL-LHC): Preliminary Design Report, CERN Yellow Reports: Monographs (CERN, Geneva, 2015).

[15] F. Zimmermann, HE-LHC and FCC-eh-CDR plan and status, in Proceedings of FCC Week 2017, Berlin, Germany (FCC Collaboration, Berlin, 2017).
[16] S. Fartoukh, Achromatic telescopic squeezing scheme and application to the LHC and its luminosity upgrade, Phys. Rev. ST Accel. Beams 16, 111002 (2013).

[17] L. A. Gonzalez et al., Test of FCC-hh beam screen at the ANKA beamline, in Proceedings of FCC Week 2017, Berlin, Germany (FCC Collaboration, Berlin, 2017).

[18] R. Cimino, V. Baglin, and F. Schäfers, Potential Remedies for the High Synchrotron-Radiation-Induced Heat Load for Future Highest-Energy-Proton Circular Colliders, Phys. Rev. Lett. 115, 264804 (2015).

[19] H. Akima, A new method of interpolation and smooth curve fitting based on local procedures, J. Assoc. Comput. Mach. 17, 589 (1970).

[20] V. Baglin (private communication).

[21] R. Cimino, M. Commisso, D. R. Grosso, T. Demma, V. Baglin, R. Flammini, and R. Larciprete, Nature of the Decrease of the Secondary-Electron Yield by Electron Bombardment and Its Energy Dependence, Phys. Rev. Lett. 109, 064801 (2012).

[22] R. Kersevan, Research program on the cryogenic beamvacuum of the FCC-hh, in Proceedings of the International Conference on High Energy Physics, 2016 (University of Chicago, Chicago, 2016).

[23] N. Kos, CERN Report No. LHC-VSS-EC-0010 version 1.0, EDMS Document No. 98531, 2004.

[24] V. V. Anashin et al., CERN technical report, Vacuum Technical Note No. 99-06, 2005.

[25] V. V. Anashin, I. R. Collins, N. V. Fedorov, B. G. Goldenberg, Oswald Gröbner, O.B. Malyshev, and V. P. Nazmov, Reflection of photons and azimuthal distribution of photoelectrons in a cylindrical beam pipe, Nucl. Instrum. Methods Phys. Res., Sect. A 448, 76 (2000).

[26] N. Mahne, V. Baglin, I. R. Collins, A. Giglia, L. Pasquali, M. Pedio, S. Nannarone, and R. Cimino, Photon reflectivity distributions from the LHC beam screen and their implications on the arc beam vacuum system, Appl. Surf. Sci. 235, 221 (2004).

[27] H. Maury Cuna and F. Zimmermann, CERN Technical Report No. CERN-ATS-Note-2011-129 TECH, 2011.

[28] R. de Maria, HL-LHC version 1.1, Collision sFlat.

[29] R. de Maria, HL-LHC version 1.1, Presqueeze optics.

[30] M. Benedikt and F. Zimmermann, Towards future circular colliders, J. Korean Phys. Soc. 69, 893 (2016).

[31] M. Benedikt, D. Schulte, and F. Zimmermann, Optimizing integrated luminosity of future hadron colliders, Phys. Rev. ST Accel. Beams 18, 101002 (2015).

[32] FCC study Web site, https://fcc.web.cern.ch/. 